\title{
Síndrome paraneoplásico de Lambert-Eaton en paciente con cáncer metastásico diseminado
}

\author{
Paraneoplastic Lambert-Eaton syndrome in a patient with disseminated metastatic cancer
}

Gregorio Arellano-Aguilar', Erik Santiago Núñez-Mojica², José Luis Gutiérrez-Velazco² y Luis Gerardo

Domínguez-Carrillo ${ }^{3 *}$

${ }^{1}$ División de Medicina; ${ }^{2}$ Interno de pregrado. Hospital Ángeles León; ${ }^{3}$ Medicina de Rehabilitación, Facultad de Medicina, Universidad de Guanajuato. León, Gto., México

\section{Resumen}

Antecedentes: Los síndromes paraneoplásicos neurológicos son poco frecuentes; se presentan en el $0.01 \%$ de todos los pacientes con cáncer. Uno de ellos es el síndrome de Lambert-Eaton, correspondiendo a un trastorno presináptico autoinmunitario de transmisión neuromuscular caracterizado por debilidad muscular y disfunción neurovegetativa, y asociado con frecuencia al carcinoma microcítico de pulmón. Caso clínico: Mujer de 72 años, con antecedentes heredofamiliares de cáncer de pulmón y leucemia, con tos seca de 7 meses, 3 meses con debilidad de los músculos de cintura pélvica, disfagia al inicio de la deglución, xerostomía, estreñimiento crónico y pérdida de peso de $10 \mathrm{~kg}$. A la exploración física: paciente postrada; examen clínico muscular: músculos de cintura pélvica en -3/5, resto en -4/5; reflejos disminuidos. Pruebas de laboratorio indicando hipercalcemia y paratohormona normal. Estudio electrofisiológico y anticuerpos anticanales de calcio positivos, demostrando síndrome de Lamber-Eaton, y estudios de imagen con afectación neoplásica en cerebro, hígado y riñón, sin lograr especificar el origen primario.

PALABRAS CLAVE: Síndrome neurológico paraneoplásico. Síndrome de Lambert-Eaton.

\begin{abstract}
Background: Neurological paraneoplastic syndromes are rare, occur in $0.01 \%$ of all cancer patients; like part of them, the Lambert-Eaton syndrome is an autoimmune presynaptic disorder of neuromuscular transmission characterized by muscle weakness and neurovegetative dysfunction, and often associated with small cell lung cancer. Case report: $A 72$ years old female with a family history of lung cancer and leukemia, with 7 months of dry cough and 3 months with waist and pelvic muscle weakness, oropharyngeal dysphagia, dry mouth, chronic constipation and weight loss of $10 \mathrm{~kg}$. Physical examination: patient prostrated; clinical muscle examination: pelvic muscles waist $-3 / 5$ and $-4 / 5$ the rest; diminished reflexes. Laboratory normal parathormone and hypercalcemia. With electrophysiological study and positive anti-voltage-gated calcium channel antibodies, confirming Lambert-Eaton syndrome and imaging studies with neoplastic condition in brain, liver and kidney, with unspecified primary origin.
\end{abstract}

KEY WORDS: Paraneoplastic neurologic syndrome. Lambert-Eaton syndrome.

\author{
Correspondencia: \\ *Luis Gerardo Domínguez Carrillo \\ Calzada de Los Paraísos, 701 \\ Col. Los Paraísos \\ C.P. 37320, León, Gto., México \\ E-mail: Igdominguez@ hotmail.com
}

Fecha de recepción: 30-09-2016

Fecha de aceptación: 14-02-2018

DOI://dx.doi.org/10.24875/CIRU.M18000006
Cir Cir. 2018;86:79-83

Disponible en PubMed www.cirugiaycirujanos.com 


\section{Introducción}

Los síndromes paraneoplásicos neurológicos (SPN) son poco frecuentes; se presentan en el $0.01 \%$ de todos los pacientes con cáncer. La primera descripción de un SPN se remonta a 1890, por el médico francés M. Auche'. Se presenta asociado a cáncer, pero no es originado por invasión directa del tumor, por metástasis ni como consecuencia del tratamiento. Los SPN pueden afectar cualquier área del sistema nervioso, ya sea central, periférico o autonómico, y pueden preceder o seguir al diagnóstico de cáncer. En ocasiones el cáncer primario no es encontrado, ni siquiera en la autopsia. Los SPN ${ }^{2}$ tienen una etiopatogenia de tipo autoinmunitario, ya que cuando el organismo trata de eliminar las células tumorales produce una respuesta inmunitaria mediada por anticuerpos o por células $T$. Los anticuerpos reaccionan con membranas accesibles, siendo directamente la causa de la enfermedad, como en los casos de receptores de acetilcolina en la miastenia grave, o en los canales de calcio abiertos por voltaje, como en el síndrome de Lambert-Eaton (SLE). El cáncer puede ser asintomático al momento de la presentación del SPN ${ }^{3}$. Algunos de los anticuerpos paraneoplásicos están específicamente asociados con cáncer, y otros no. La incidencia y la prevalencia exactas de los SPN es desconocida; se ha estimado que el $0.5-1 \%$ de todos los pacientes con cáncer presentan SPN clínicamente detectado 4 . Cuando el SPN afecta a la unión neuromuscular, el síndrome neurorológico más común es miastenia grave, que se asocia en el $15 \%$ de los pacientes a timoma, siguiéndole en frecuencia el SLE, que afecta al $3 \%$ de los pacientes con cáncer pulmonar microcítico.

Al presentarse una paciente en la que se llegó al diagnóstico de SLE, realizamos esta comunicación.

\section{Caso clínico}

Mujer de 72 años, ama de casa, originaria y residente de León, Guanajuato, con antecedentes heredofamiliares de importancia: madre finada por cáncer de pulmón, hermano con leucemia sin especificar el tipo, un hijo con espondilitis anquilosante. Hipertensión arterial de más de 10 años controlada con inhibidor de la enzima convertidora de angiotensina. Gesta 4, para 4. Con padecimiento actual con evolución de 7 meses, caracterizado por tos seca; 3 meses después se agregan fatiga y debilidad primordialmente en músculos de cintura y pélvica, modificándose a generalizada en los últimos 2 meses y presentando disfagia al inicio de la deglución, además de sequedad de boca y estreñimiento crónico, con evacuación cada 4 días; relata pérdida de peso de $10 \mathrm{~kg}$ en los últimos 4 meses atribuida a la disfagia, y anhedonia. La debilidad progresiva la ha llevado a la postración, actualmente requiere ayuda para cambio de posición de sedente a bipedestación, puede caminar en casa con ayuda de +++. A la exploración física: presión arterial 110/70 mmHg; frecuencia cardiaca 106 lpm; frecuencia respiratoria $22 \mathrm{rpm}$; temperatura $36.8{ }^{\circ} \mathrm{C}$; $\mathrm{SatO}_{2} 89 \%$. Paciente postrada, marcha no valorable, alerta con desorientación temporal, con lentitud en procesamiento de información; pares craneales normales, fondos de ojos normales. Tórax: simétrico, con presencia de tiros intercostales, ampliación y amplexación disminuidas; ruidos respiratorios con murmullo vesicular normal, ruidos cardiacos rítmicos, abdomen blando sin visceromegalias. Examen clínico muscular: músculos de cintura pélvicas y escapular en - $3 / 5$, resto en $-4 / 5$, reflejos osteotendinosos disminuidos; pulsos y llenado capilar normales. Se hospitalizó para su estudio, con pruebas de laboratorio que mostraron: hemoglobina $10.2 \mathrm{~g} / \mathrm{dl}$; hematocrito $32.1 \%$; leucocitosis

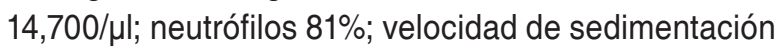
globular $33 \mathrm{~mm} / \mathrm{h}$; plaquetas $475,000 / \mu \mathrm{l}$; calcio $12.2 \mathrm{mg} / \mathrm{dl}$; potasio $5.7 \mathrm{mmol} / \mathrm{l}$; proteínas totales $5.7 \mathrm{~g} /$ dl; albúmina $2.5 \mathrm{~g} / \mathrm{dl}$; globulina $3.2 \mathrm{~g} / \mathrm{dl}$; calcio corregido por albúmina $13.6 \mathrm{mg} / \mathrm{dl}$; bilirrubinas normales; fosfatasa alcalina $188 \mathrm{U} / \mathrm{l}$; deshidrogenasa láctica 684 U/l; glucosa $178 \mathrm{mg} / \mathrm{dl}$; urea $68.5 \mathrm{mg} / \mathrm{dl}$; creatinina $1.2 \mathrm{mg} / \mathrm{dl}$; creatina fosfocinasa $25 \mathrm{U} / \mathrm{l}$; paratohormona intacta $38.8 \mathrm{pg} / \mathrm{ml}$; EGO: pH 5, hemoglobina ++, nitritos positivo, leucocitos $>100$, eritrocitos $2-4$ y bacterias abundantes. Con los datos anteriores se establecieron los diagnósticos de infección de vías urinarias, hiperglucemia, hipercalcemia, anemia normocítica normocrómica de grado II, posible neoplasia maligna de localización por determinar, y probable SLE. Se solicitaron resonancia magnética de cráneo y tomografía de tórax y abdomen (Figs. 1 a 3), encontrando infiltraciones metastásicas múltiples (sin poder especificar su origen), así como electromiografía de fibra única con resultados que confirmaron la alteración de placa neuromuscular, por lo que se efectuó una prueba de estimulación nerviosa repetitiva que mostró, con estimulación de baja frecuencia, una respuesta de amplitud decreciente de los potenciales, los cuales se incrementaron con estimulación de alta frecuencia, corroborando electrofisiológicamente el SLE. Al 


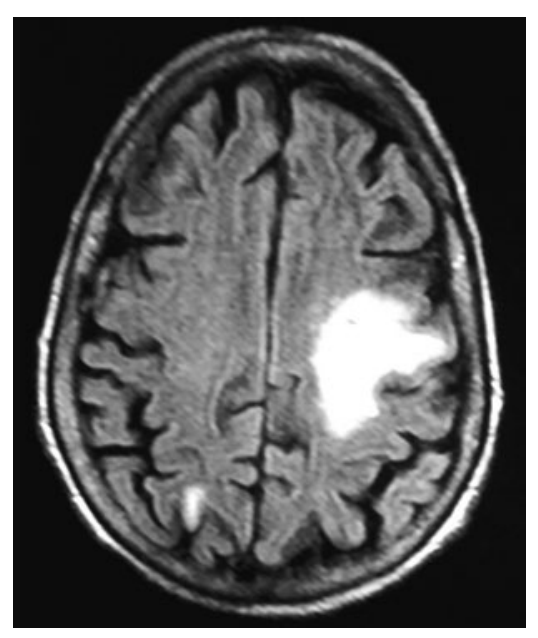

Figura 1. Resonancia magnética de cráneo en corte axial, mostrando mayor amplitud del espacio subaracnoideo de manera difusa y extensa zona de gliosis (Fasekas III), amorfas de aspecto confluente, en la sustancia blanca subcortical del lóbulo frontal izquierdo.

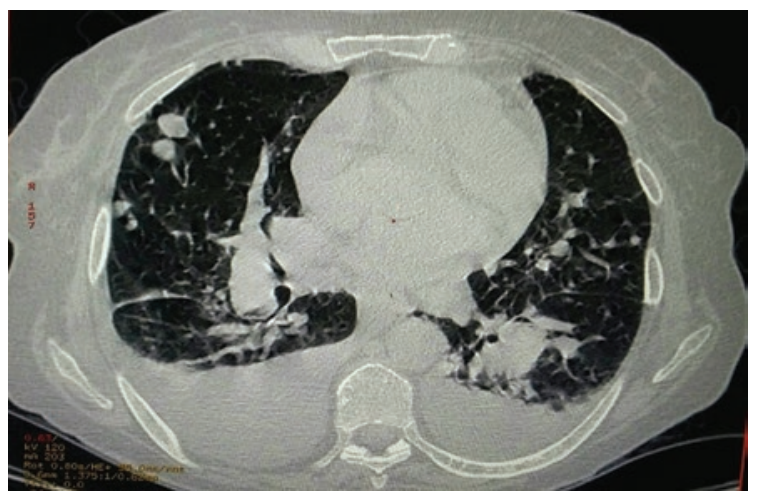

Figura 2. Imagen de tomografía de tórax en corte axial, mostrando imágenes nodulares distribuidas al azar, engrosamiento de las paredes bronquiales e infiltrado micronodular y derrame pleural bilateral.

comentar los diagnósticos con la paciente y sus familiares, se propuso realizar gastrostomía para alimentación enteral, estudio por oncología y manejo inicial con esteroides. Se inició tratamiento con gentamicina, controlando la infección urinaria; la paciente permaneció 12 días en hospitalización bajo tratamiento con 3,4-diaminopiridina fosfato a dosis de $15 \mathrm{mg}$ tres veces al día, prednisona a dosis de $10 \mathrm{mg} / \mathrm{kg}$ de peso y oxígeno a $2 \mathrm{l} / \mathrm{min}$, presentando mejoría en la deglución, incorporarse por si sola y realizando marcha con asistencia de andador. Fue canalizada a oncología para su estudio y seguimiento. Se anota que se envió muestra a laboratorio especializado solicitando determinación de anticuerpos contra los canales de calcio dependientes del voltaje (VGCC) y anticuerpos contra los receptores de acetilcolina. Los resultados se recibieron 15 días y mostraron VGCC 85 pmol/l (normal menos de $20 \mathrm{pmol} / \mathrm{l}$ ) y negativo para anticuerpos contra los receptores de acetilcolina, con lo que se confirmó el diagnóstico.

\section{Discusión}

En el caso que nos ocupa, la historia familiar de dos variedades de cáncer, aunada a las manifestaciones de fatiga muscular inicialmente en la cintura pélvica, la hiporreflexia y las manifestaciones neurovegetativas, como sequedad de boca y estreñimiento, así como la baja importante en el peso corporal, orientaron a la posibilidad de malignidad, apoyados en la hipercalcemia con la paratohormona normal, sospechando que las manifestaciones musculares correspondieran al SLE. Los estudios de imagen corroboraron la presencia de neoplasia (aún sin determinar su origen) con afectación del sistema nervioso central, el hígado y el riñón, además de que la prueba de electrofisiología apoya la presencia del SLE.

EI SLE es un trastorno presináptico autoinmunitario de transmisión neuromuscular caracterizado por debilidad muscular y disfunción neurovegetativa. Se ve asociado con frecuencia al carcinoma microcítico de pulmón. El primer caso reportado pertenece a Anderson, et al. ${ }^{5}$ en 1953, y posteriormente Lambert, Eaton y Rooke, en 1957, reportan un informe de seis pacientes con transmisión neuromuscular defectuosa asociada a neoplasia y describen las características clínicas y electrofisiológicas del síndrome miasténico que lleva sus apellidos ${ }^{6}$.

La prevalencia ${ }^{7}$ del SLE estimada en todo el mundo está entre 1/250,000 y 1/333,300. El inicio de la enfermedad suele presentarse alrededor de los 40 años, pero puede ocurrir a cualquier edad. Se caracteriza por la tríada clínica de debilidad muscular proximal, trastornos neurovegetativos y disminución de los reflejos tendinosos ${ }^{8}$ (todos presentes en el caso). Los músculos faríngeos se ven involucrados en el SLE solo en el $7 \%$ de los pacientes, presentando disfagia ${ }^{8}$ (como en este caso). Las neoplasias, primordialmente el carcinoma microcítico de pulmón, están presentes en el $50-60 \%$ de los casos de SLE. Puede verse asociada a ataxia cerebelosa, en cuyo caso casi siempre está acompañada de carcinoma microcítico de pulmón. Hasta el $90 \%$ de los pacientes con SLE presentan anticuerpos del tipo inmunoglobulina $G$ contra el canal de calcio regulado por voltaje de tipo P/Q19; la disfunción o la reducción de la cantidad de estos canales inhibe la liberación de acetilcolina de la placa terminal presináptica, lo que provoca una disminución 

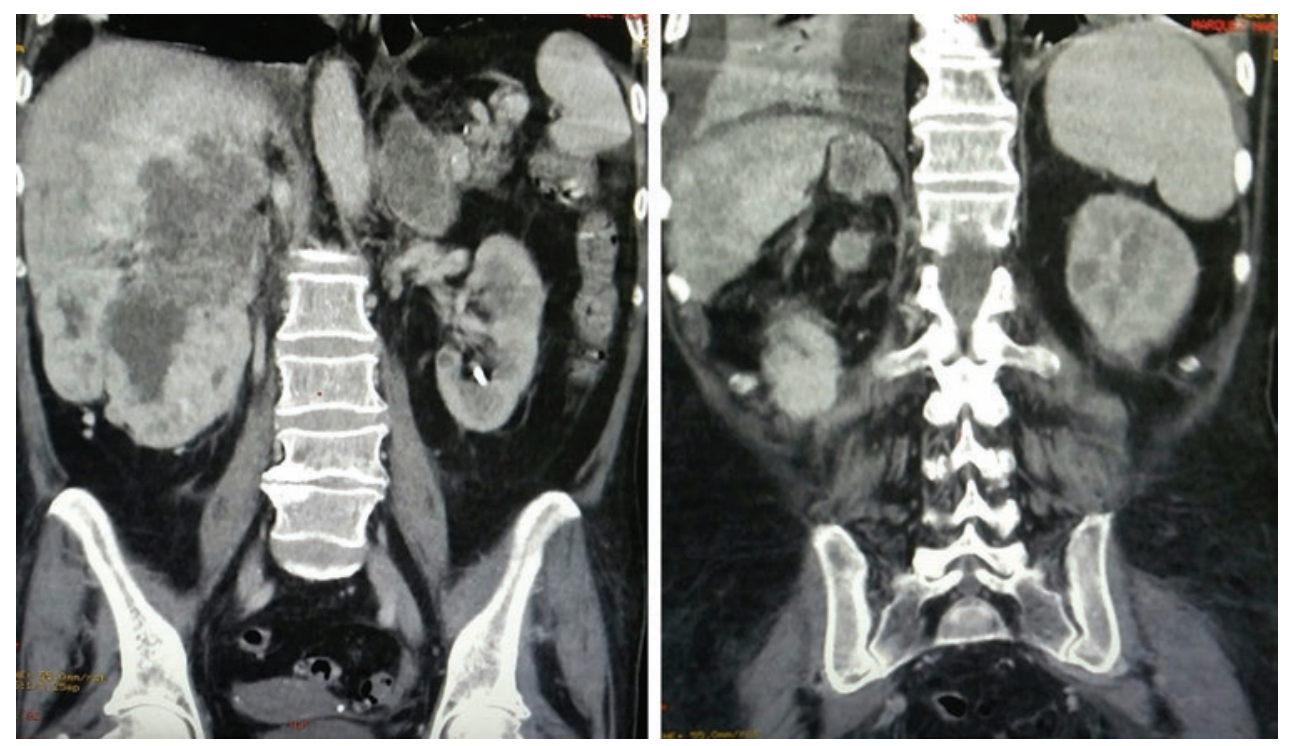

Figura 3. Imágenes tomográficas en corte coronal, mostrando una lesión tumoral en el polo superior del riñón derecho, con dimensiones $11.2 \times 10.8 \mathrm{~cm}$, de aspecto heterogéneo con zonas hipodensas, en contacto con el lóbulo hepático derecho, que muestra infiltración tumoral. La glándula suprarrenal izquierda mide $4.1 \times 3.8 \mathrm{~cm}$ y es hipodensa, de aspecto nodular.

de la transmisión neuromuscular y la consiguiente debilidad de los músculos. El anticuerpo VGCC se encuentra en casi el $100 \%$ de los casos de SLE, y no difiere entre las formas paraneoplásicas y no paraneoplásicas. Por otra parte, se menciona la asociación entre el SLE, neoplásico o no, con el antígeno principal de histocompatibilidad HLA-B8, siendo más fuerte la asociación con la forma no neoplásica. Actualmente se describe un anticuerpo sérico, denominado $S O X 1^{10}$, en el SLE y el carcinoma microcítico de pulmón, presente en el $64 \%$ de los casos, teniendo una especificidad del $95 \%$ y una sensibilidad moderada del $65 \%$.

Además de la tríada clínica clásica (si bien no siempre están presentes los tres síntomas), el diagnóstico del SLE se basa en la detección de anticuerpos VGCC mediante ensayos de radioinmunoprecipitación, así como en las anomalías típicas en el test de la estimulación nerviosa repetitiva ${ }^{11}$, caracterizadas por baja amplitud del potencial de acción muscular compuesto, además de un decremento paulatino ante la estimulación eléctrica con bajas frecuencias, que se ve invertida con el aumento de la amplitud del potencial de acción a la estimulación eléctrica de alta frecuencia o tras un breve ejercicio (facilitación posejercicio). La electromiografía de fibra única puede confirmar un trastorno de la unión neuromuscular, pero es inespecífica. El diagnóstico del SLE precede casi de manera invariable al descubrimiento de un carcinoma microcítico de pulmón; sin embargo, puede asociarse a otras neoplasias, como trastornos linfoproliferativos, cáncer de mama, colon, estómago, vesícula biliar, riñones y vejiga, adenocarcinoma de pulmón, páncreas y próstata, así como carcinoide intratorácico. Los pacientes con síndrome miasténico, de manera simultánea, tienen una enfermedad autoinmunitaria en alrededor del $25-45 \%$ de los casos, con presencia de autoanticuerpos específicos de órgano. La prevalencia de estos autoanticuerpos es más alta en ausencia de un carcinoma subyacente ${ }^{12}$. Clínicamente y serológicamente, el $30 \%$ de los casos se presentan sin tumor identificable. El reconocimiento de un SPN clásico asociado con un anticuerpo único es más una excepción que una regla.

En el diagnóstico diferencial deben incluirse la miastenia grave, la miositis por cuerpos de inclusión, el síndrome de Guillain-Barré, la esclerosis lateral amiotrófica, la estenosis del canal lumbar, la enfermedad de Parkinson en fase temprana y el parkinsonismo de la mitad inferior del cuerpo.

El manejo debe considerar en primer término el tratamiento de la neoplasia, si la hubiese, de tal modo que la debilidad puede mejorar tras una terapia efectiva del cáncer. No existe tratamiento curativo del SLE, siendo fundamentalmente sintomático ${ }^{13}$. Esto incluye la 3,4-diaminopiridina fosfato, cuyo mecanismo de acción es el bloqueo selectivo de los canales de potasio en la membrana presináptica, buscando una mayor duración del potencial de acción presináptico por incremento de la liberación de acetilcolina desde la terminal colinérgica y adrenérgica. Clínicamente, la 3,4-diaminopiridina disminuye la debilidad muscular y los síntomas de disfunción autonómica hasta en un 
$85 \%$ de los pacientes. Si el tratamiento sintomático es insuficiente, una terapia inmunosupresora con prednisona, sola o en combinación con azatioprina, puede producir un control a largo plazo de la enfermedad. La plasmaféresis y la administración de altas dosis de inmunoglobulinas intravenosas tienen un efecto a corto plazo. Los anticuerpos monoclonales, como el rituximab ${ }^{14}$, han empezado a utilizarse con resultados aparentemente promisorios. Es imprescindible llevar a cabo un tratamiento eficaz contra cualquier tumor presente, tanto para controlar dicho tumor como para mejorar los síntomas clínicos del SLE. En general, el SLE responde bien a los tratamientos sintomáticos e inmunosupresores. No obstante, puede afectar a las actividades diarias y a la calidad de vida de los pacientes. La esperanza de vida depende de la presencia del cáncer de pulmón. Sin cáncer, la esperanza de vida se considera normal ${ }^{15}$. Como el carcinoma microcítico de pulmón es un cáncer muy agresivo, el pronóstico de los pacientes con SLE y carcinoma microcítico de pulmón suele ser malo. La supervivencia media es de entre 17 y 24 meses, aunque la cantidad de pacientes con remisión de larga duración o con curación es de aproximadamente el $20 \%$, en comparación con menos del $2 \%$ de los pacientes con carcinoma microcítico de pulmón sin SLE.

\section{Conclusiones}

Se trata de una paciente con cáncer metastásico, debutante como SPN y SLE, que permaneció 12 días hospitalizada y presentó mejoría, pero por las características de diseminación de su cáncer presenta pronóstico malo a corto plazo.

\section{Responsabilidades éticas}

Protección de personas y animales. Los autores declaran que para esta investigación no se han realizado experimentos en seres humanos ni en animales.

Confidencialidad de los datos. Los autores declaran que han seguido los protocolos de su centro de trabajo sobre la publicación de datos de pacientes.

Derecho a la privacidad y consentimiento informado. Los autores han obtenido el consentimiento informado de los pacientes y/o sujetos referidos en el artículo. Este documento obra en poder del autor de correspondencia.

\section{Financiamiento}

Los autores declaran no haber recibido ningún tipo de financiación.

\section{Conflicto de intereses}

Los autores declaran que no existe conflicto de intereses.

\section{Bibliografía}

1. Auche M. Des nevrites peripheriques chez les cancereux. Rev Med. 1890;10:785-807.

2. Grausa F, Dalmaua J. Paraneoplastic neurological syndromes Curr Opin Neurol. 2012;25:795-801.

3. Höftberger R, Rosenfeld MR, Dalmau J. Update on neurological paraneoplastic syndromes. Curr Opin Oncol. 2015;27:489-95.

4. Braik T, Evans AT, Telfer M, McDunn S. Paraneoplastic neurological syndromes: unusual presentations of cancer. A practical review. Am J Med Sci. 2010;340:301-8.

5. Anderson HJ, Churchill DH, Richardson AT. Bronchial neoplasm with myasthenia, prolonged apnea after administration of succinylcholine. Lancet. 1953;265:1291-3.

6. Eaton LM, Lambert EH. Electromyography and electrical stimulation of nerves in disease of the motor unit: observations on a myasthenic syndrome associated with malignant tumors. JAMA. 1957;163:1117-24.

7. Wirtz PW, Nijnuis MG, Sotodeh M, et al. The epidemiology of myasthenia gravis, Lambert-Eaton myasthenic syndrome and their associated tumours in the northern part of the province of South Holland. J Neurol. 2003;250:698-701.

8. Fernández-Torrón R, Arcocha J, López-Picazo JM, et al. Isolated dysphagia due to paraneoplastic myasthenic syndrome with anti-P/Q-type voltage-gated calcium-channel and anti-acetylcholine receptor antibodies. Neuromuscul Disord. 2011;21:126-8.

9. Motomura M, Lang B, Johnston I, et al. Incidence of serum anti-P/Qtype and anti-N-type calcium channel autoantibodies in the Lambert-Eaton myasthenic syndrome. J Neurol Sci. 1997;147:35-42.

10. Titulaer MJ, Klooster R, Potman M, et al. SOX antibodies in small-cell lung cancer and Lambert-Eaton myasthenic syndrome: frequency and relation with survival. J Clin Oncol. 2009;27:4260-7.

11. AAEM Quality Assurance Committee. Practice parameter for repetitive nerve stimulation and single fiber EMG evaluation of adults with suspected myasthenia gravis or Lambert-Eaton myasthenic syndrome: summary statement. Muscle Nerve. 2001;24:1236-8.

12. Pellkofer HL, Armbruster L, Krumbholz M, et al. Lambert-Eaton myasthenic syndrome differential reactivity of tumor versus non-tumor patients to subunits of the voltage-gated calcium channel. J Neuroimmunol. 2008; 204:136-9.

13. Skeie GO, Apostolski S, Evoli A, et al. Guidelines for treatment of autoimmune neuromuscular transmission disorders. Eur J Neurol. 2010; 17:893-902.

14. Maddison P, McConville J, Farrugia ME, et al. The use of rituximab in myasthenia gravis and Lambert-Eaton myasthenic syndrome. J Neurol Neurosurg Psychiatry. 2011;82:671-3.

15. Wirtz PW, Smallegaange TM, Wintzen AR, et al. Difference in clinical features between the Lambert-Eaton myasthenic syndrome with and without cancer: an analysis of 227 published cases. J Neurol Neurosurg Psychiatry. 2002;104:359-63. 